
\title{
3 Research Square \\ First-Principles Study of The Structural Phase Transition Process of Solid Nitrogen Under Pressure
}

\section{Zhi-Xin Bai}

Southwest Jiaotong University

Cheng-Lu Jiang

Southwest Jiaotong University

\section{Sheng-Hai Zhu}

Southwest Jiaotong University

Mi Zhong

Southwest Jiaotong University

Ming-Jian Zhang

Southwest Jiaotong University

Fu-Sheng Liu

Southwest Jiaotong University

Bin Tang

Northwestern Polytechnical University

Qi-Jun Liu

Southwest Jiaotong University

Xianghui Chang ( $\nabla$ xianghuichang@swjtu.edu.cn)

Southwest Jiaotong University https://orcid.org/0000-0003-2638-747X

\section{Research Article}

Keywords: solid nitrogen, acoustic phonons, structural phase transition, high pressure

Posted Date: July 7th, 2021

DOl: https://doi.org/10.21203/rs.3.rs-670245/v1

License: (c) (i) This work is licensed under a Creative Commons Attribution 4.0 International License. Read Full License 


\section{Abstract}

Due to the diversity of solid nitrogen structure, its phase transition has been a hot topic for many scientists. Herein, we first studied the structural softening of rhombohedral solid nitrogen under pressure using first-principles calculations. Then, a new criterion, Egret criterion, was proposed to predicting the whole process from beginning to end of structural phase transition of solid nitrogen. Based on the discussion of acoustic phonons, we concluded that the phase transition of rhombohedral solid nitrogen starts from $k$-point $F$ along the $[-1,-1,0]$ direction in a-axis, and the structural phase transition velocity is slow. Also, we use the Egret criterion proposed by us to predict the emergence of $\xi-\mathrm{N}_{2}$ and the stability of $\xi-\mathrm{N}_{2}$ at $17 \mathrm{GPa}$ and $22 \mathrm{GPa}$, respectively, and this result is in good agreement with the phase diagram of nitrogen.

\section{Introduction}

Nitrogen molecule undergoes many structural phase transitions at different temperatures and pressures. At least five different phases have been found for the nitrogen molecule under pressure of less than 20 GPa: cubic a- $\mathrm{N}_{2}(\mathrm{~Pa} 3)$ [1], tetragonal $\gamma-\mathrm{N}_{2}\left(\mathrm{P} 4{ }_{2} / \mathrm{mnm}\right)$ [1], rhombohedral $\varepsilon-\mathrm{N}_{2}(\mathrm{Rc})[2,3]$, hexagonal $\beta-\mathrm{N}_{2}$ $\left(\mathrm{P}_{3} / \mathrm{mmc}\right)[4,5]$ and cubic $\delta-\mathrm{N}_{2}(\mathrm{Pm} 3 \mathrm{n})[6-8]$. In recent years, many polymeric phases for solid nitrogen also have been obtained, such as cubic gauche nitrogen (cg-N) [9-12], layered-polymeric nitrogen (LP-N) $[13,14]$, hexagonal layered polymeric nitrogen (HLP-N) [14] and post-layered-polymeric nitrogen (PLP-N) [15].

Different molecular and polymeric phases of nitrogen are different in their physical and chemical properties and have a wide range of applications, especially the application demand of rich nitrogen in high energy density materials [16]. This peculiarity of nitrogen has received extensive attention from many researchers. Therefore, the crystal structure transition [17-25], structure transition path [26], magnetic transition [27] and polarization symmetry [28] and other related contents of nitrogen have been studied extensively in the experimental and theoretical aspects. With the development of computer simulation, first-principles calculation plays an irreplaceable role in material research, and many key properties of materials have been accurately predicted. Recently, Jiang et al. [29] first proposed the view that phonon group velocity with negative value is the structural phase transition velocity. The negative phonon group velocity refers to the plastic stretching or compression velocity in a certain direction, that is, it represents the phase transition of the structure [29]. This study shows that the change of structural phase transition velocity of rhombohedral solid nitrogen under pressure is regular. Therefore, we wonder if the phonon group velocity can be used to predict the whole process from beginning to end of structural phase transition.

In this paper, we first studied the softening of rhombohedral solid nitrogen before the phase transition based on the first-principles calculation, and then verified our question and proposed a new criterion for predicting the whole process from beginning to end of structural phase transition named Egret criterion. 


\section{Computational Methods}

In this study, the first-principles calculations were performed using the norm-conserving pseudopotential method based on the density functional theory as implemented in the CASTEP [30-33]. The generalized gradient approximation (GGA) $[30,31]$ with PBESOL [34] functional was adopted. The cutoff energy of $770 \mathrm{eV}$ and the Monkhorst Pack mesh of $3 \times 3 \times 3$ were selected. Moreover, the geometrical structure in this paper was allowed to relax until the total energy was less than $5.0 \times 10^{-6} \mathrm{eV} /$ atom. The maximum force, maximum stress, and maximum displacement were set to $0.01 \mathrm{eV} / \AA \AA 0.02 \mathrm{GPa}$ and $5.0 \times 10^{-4} \AA$, respectively.

\section{Results And Discussion}

\subsection{Electronic properties}

The computational model is rhombohedral solid nitrogen with space group $\mathrm{R} \overline{3} \mathrm{C}$, as shown in Fig.1. The nitrogen atoms of the primitive cell structure have two different positions, namely N1 and N2 in the Fig.1.The calculated structural parameters along with the experimental data [3] of solid nitrogen are shown in Fig.2. Lattice parameters decrease with the increase of pressure, and the results we obtained based on GGA calculations with PBESOL are in good agreement with the experimental data at $7.8 \mathrm{GPa}$ [3]. Figure3 and Fig.4 show the variation of the band gap under pressure. It is found that the band gap gradually decreases under pressure. The reason for this phenomenon is that the pressure leads to the increase of matrix concentration [29]. From Fig.3, we could easily see that the energy level at the bottom of the conduction band decreases under pressure, which makes it easier for electrons in the valence band to transit to the conduction band, making it easier to conduct electricity. The total and partial densities of states are shown in Fig.5. From the figure, the energy level of electronic state decreases under pressure and the conduction band part of solid nitrogen is mainly composed of $\mathrm{N}-2 \mathrm{p}$ electronic states.

\subsection{The softening of solid nitrogen before structural phase transition}

In this paper, we aim to clarify the structural softening of solid nitrogen before the phase transition. Obviously, we cannot judge the stability of the structure by analysing the above electronic properties. So, we must study from the aspect of phonons. The phonon dispersion curves along the high-symmetry direction are calculated. The acoustic phonons are selected for discussion to investigate the structural phase transition, as shown in Fig. 6 . In order to understand the changes of acoustic phonons under pressure more intuitively, the three acoustic phonons of solid nitrogen under pressure (shown in Fig. 6(a)) are divided into acoustic phonons in a-axis, b-axis and c-axis, as shown in Fig. 6(b), Fig. 6(c) and Fig. $6(\mathrm{~d})$, respectively. From Fig. 6(a), the virtual frequency appears at the pressure of $16.5 \mathrm{GPa}$, that is, the structure of solid nitrogen begins to undergo phase transition between $16 \mathrm{GPa}$ and $16.5 \mathrm{GPa}$. Figure $6(\mathrm{~b})$, Fig. $6(\mathrm{c})$ and Fig. $6(\mathrm{~d})$ show that the acoustic phonon energies at k-point $F(0.5,0.5,0)$ in a-axis are negative, while the acoustic phonon energies at k-point $\mathrm{F}$ in b-axis and c-axis are positive. The acoustic 
phonon energies at k-point $Z(0.5,0.5,0.5)$ in a-axis, $b$-axis and c-axis are negative, meanwhile, the values of acoustic phonon energies in these three axes are the same under the corresponding pressure.

As we all know, the presence of virtual frequencies in acoustic phonons indicates structural instability, that is, negative acoustic phonons symbolize a structural phase transition. Jiang et al. [29] proposed for the first time the idea that negative phonon group velocity is the structural phase transition velocity. Based on this view, we used following formula $[29,35,36]$ to calculate the phonon group velocity of rhombohedral solid nitrogen under pressure:

$E=4.192 \times 10^{-4} \times v_{p} \times Q_{\max } \times \operatorname{Sin} \frac{\pi}{4}$

where $E$ is acoustic phonon energy, $v_{p}$ is the phonon group velocity, $Q_{\max }$ is the boundary length of Brillouin region and $\operatorname{Sin} \frac{\pi}{4}$ is the average energy of the elastic wave. Table 1 lists the calculated negative group velocity and positive group velocity of solid nitrogen. We found that there are negative phonon group velocities at k-point $F$ from $F$ to $G$ in a-axis and at k-point $Z$ from $G$ to $Z$ in a, b, c-axis. At $16.5 \mathrm{GPa}$, the phonon group velocity at k-point $F$ from $F$ to $G$ in a-axis is $-360.0 \mathrm{~m} / \mathrm{s}$, while the phonon group velocities at k-point in other directions at this pressure are all positive. Negative phonon group velocity is the structural phase transition velocity [29]. Therefore, we concluded that the phase transition of rhombohedral solid nitrogen starts from k-point $F$ along the $[-1,-1,0]$ direction, and the structural phase transition velocity is slow. At $18 \mathrm{GPa}$, the phonon group velocity at k-point $Z$ from $\mathrm{G}$ to $Z$ in $a, b, c$-axis also becomes negative, which is $-258.4 \mathrm{~m} / \mathrm{s}$. In other words, starting from $18 \mathrm{GPa}$, the k-point $Z$ of rhombohedral solid nitrogen also begins to undergo phase transition along the direction of $[1,1,1]$, and the structural phase transition speed is slow. 
Table 1

The negative and positive phonon group velocities of rhombohedral solid nitrogen under pressure.

\begin{tabular}{|c|c|c|c|c|c|c|}
\hline \multirow[t]{3}{*}{$\begin{array}{l}\text { Pressure } \\
(\mathrm{GPa})\end{array}$} & \multicolumn{2}{|c|}{$\begin{array}{l}\text { Negative group velocity } \\
\text { (phase transition velocity) }\end{array}$} & \multicolumn{4}{|c|}{ Positive group velocity } \\
\hline & $F(F$ to $G)$ & $\mathrm{Z}$ (G to Z) & $F(F$ to $C$ & & & $\mathrm{Z}(\mathrm{G}$ to $\mathrm{Z})$ \\
\hline & a-axis & $a, b, c$-axis & a-axis & b-axis & c-axis & a, b. c-axis \\
\hline 5 & & & 412.6 & 940.6 & 1038.5 & 384.7 \\
\hline 10 & & & 1323.6 & 1747.7 & 1862.7 & 1046.6 \\
\hline 15 & & & 945.2 & 1677.6 & 2066.7 & 793.4 \\
\hline 16 & & & 625.0 & 1584.5 & 2070.1 & 631.0 \\
\hline 16.5 & -360.0 & & & 1521.5 & 2067.2 & 519.0 \\
\hline 17 & -347.2 & & & 1483.9 & 2063.2 & 374.4 \\
\hline 18 & -732.0 & -258.4 & & 1379.1 & 2069.2 & \\
\hline 19 & -958.5 & -786.0 & & 1204.8 & 1830.6 & \\
\hline 20 & -982.4 & -903.4 & & 1260.4 & 1926.9 & \\
\hline 21 & -1090.2 & -969.1 & & 1243.2 & 1966.0 & \\
\hline 22 & -1311.1 & -612.8 & & 1070.7 & 1933.2 & \\
\hline 25 & -1405.8 & -1139.9 & & 1202.8 & 2130.1 & \\
\hline 30 & -1586.3 & -1132.1 & & 1140.6 & 2077.3 & \\
\hline 35 & -1644.3 & -1158.1 & & 1271.9 & 2094.6 & \\
\hline 37 & -1686.1 & -1168.3 & & 1306.3 & 2108.6 & \\
\hline 38 & -1712.1 & -1194.9 & & 1319.5 & 2111.7 & \\
\hline 39 & -1743.9 & -1209.8 & & 1326.6 & 2118.9 & \\
\hline 40 & -1777.8 & -1225.6 & & 1331.9 & 2113.2 & \\
\hline 45 & -1994.1 & -1346.6 & & 1283.2 & 2121.7 & \\
\hline
\end{tabular}

\subsection{Mechanical properties}

It is well known that the response of a solid under pressure is related to the elastic constants, which can be used to judge the mechanical stability of a solid structure. In order to further explore the structural stability of rhombohedral solid nitrogen under pressure, the elastic constant with pressure is shown in Fig. 7. Due to the positive correlation between stress and strain, the elastic constants increase under 
pressure. At $5 \mathrm{GPa}$, the elastic constants are the smallest. In other word, the deformation resistance of rhombohedral solid nitrogen is the weakest at $5 \mathrm{GPa}$. There are turning points in the value of $\mathrm{C}_{11}, \mathrm{C}_{44}$, and $\mathrm{C}_{66}$ at $30 \mathrm{GPa}$, indicating a softening of the structure. $\mathrm{C}_{44}$ suddenly decreases at $19 \mathrm{GPa}$, meaning that the structure has softened. According to the mechanical stability criterion of rhombohedral crystal system under pressure [37]:

$$
\left\{\begin{array}{l}
C_{11}-P>0 \\
C_{11}-P-\left|C_{12}+P\right|>0 \\
C_{44}-P>0 \\
\left(C_{33}-P\right)\left(C_{11}+C_{12}\right)-2\left(C_{13}+P\right)^{2}>0 \\
\left(C_{44}-P\right)\left(C_{11}-C_{12}-2 P\right)-2 C_{14}^{2}>0
\end{array}\right.
$$

In order to more intuitively observe the mechanical stability of rhombohedral solid nitrogen, Fig. 8 was drawn. As can be seen from the figure, 25.4 GPa, 26.9 GPa and 27.6 GPa are the mechanical unstable pressures of rhombohedral solid nitrogen. It is not meet $\left(C_{44}-P\right)\left(C_{11}-C_{12}-2 P\right)-2 C_{14}^{2}>0$ at $27.6 \mathrm{GPa}$. And $c_{66}\left(C_{66}=\left(C_{11}-C_{12}\right) / 2\right)$ represents the resistance to deformation in the [110] direction. This also proves that virtual frequency appears at k-point $F$ along the direction of $[-1,-1,0]$ in a-axis obtained from our analysis of phonon spectra above.

\subsection{Egret criterion}

By observing Table 1, a very interesting phenomenon is that the phase transition velocity of rhombohedral solid nitrogen structure (the part of negative phonon group velocities) is slow at the beginning of the phase transition, and then increases and then decreases under the action of pressure. In order to observe this phenomenon more intuitively, we have drawn Fig. 9. After analysis, the changes of structural phase transition velocity from one solid nitrogen structure to another is described as follows: At the beginning of structural phase transition, the structural phase transition speed of solid nitrogen is slow. Then, under the action of pressure, it first increases and then decreases, and finally increases and tends to be stable. This change is regular. And the structural phase transition velocity is defined by negative phonon group velocity [29]. So we would use the phonon group velocity to show the whole process in structural phase transition.

For the convenience of our understanding, the phonon group velocities of rhombohedral solid nitrogen under pressure are shown in Fig. 10. First of all, before the structural phase transition (i.e. before the phonon group velocity is greater than zero), the phonon group velocity increases first and then decreases under pressure. The decrease of the phonon group velocity is the softening of the structure, which is consistent with the experimental observation of structure softening before solid-liquid transition [38]. After the structural phase transition occurs, the negative phonon group velocity first increases and then decreases under pressure. Combining the phase diagram of nitrogen [39], we considered that the $\xi$ phase of solid nitrogen appears at $17 \mathrm{GPa}$, and this phase becomes a stable phase at $22 \mathrm{GPa}$. Why did we think 
that a stable new phase appears at the turning point of the negative phonon group velocity? We made the following explanations: The process of a new phase from appearance to stability is the process of atoms moving to an equilibrium position. The original phase also exists when the new phase just appears, and there are two equilibrium positions, one is the equilibrium position of the atomic vibration of the original phase, another is the equilibrium position of the new phase vibration. The vibration of the original phase atoms in the equilibrium position will affect the vibration of the atoms that are about to reach the stable new phase (close to the equilibrium position), that is to say, the atoms have potential interaction between original phase and new phase. When a certain pressure is exceeded, the equilibrium position of the new phase will dominate. Therefore, the phonon group velocity under pressure appears a reversal point.

Based on the discussion above, we believed that it is reasonable to use the phonon group velocity to predict the whole process of the structural phase transition of the solid nitrogen structure from the beginning to the end. Therefore, we proposed a criterion for deciding the new solid nitrogen structure becoming stable under pressure, the Egret criterion, which is expressed as follows:

The change of the phonon group velocity of a solid nitrogen structure under pressure is as follows: firstly, the positive phonon group velocity decreases under pressure before the structural phase transition occurs (i.e. the softening of the solid nitrogen structure). Then the phonon group velocity of the solid nitrogen becomes negative, and the structure begins to undergo a phase transition. The negative phonon group velocity increases first and then decreases under pressure, and finally becomes stable after a slight increase under pressure. In this process, a new stable phase appears at the pressure corresponding to the reversal point of the negative phonon group velocity.

Also, we predict that this criterion can be applied to many solid structures, and our follow-up work will continue to verify it.

\section{Conclusions}

The softening process of rhombohedral solid nitrogen is investigate using First principles calculation. The structural parameters of the model we used are basically consistent with the experimental values. The calculated energy band structures and density of states change stably under pressure. The phonon spectra of rhombohedral solid nitrogen show that virtual frequency occurs between $16 \mathrm{GPa}$ and 16.5 $\mathrm{GPa}$, that is, the structure of rhombohedral solid nitrogen begins to become unstable between $16 \mathrm{GPa}$ and 16.5 GPa. We find that rhombohedral solid nitrogen phase transition starts from $\mathrm{k}$ point $\mathrm{F}$ along the $[-1,-1$, 0] direction ( $F$ to $G$ ) in a-axis, and it is verified by mechanical properties. In this paper, the most important is that we firstly put forward a new criterion for predicting the structural phase transition process of nitrogen by phonon group velocity, which provides a new perspective for experimental observation. And we predict that this criterion applies to many phase transitions of solid structures.

\section{Declarations}


1. Funding

This work was supported by the Fund of the Key Laboratory of National Defense Science and Technology (Grant No. 6142A03182008).

2. Conflicts of interest/Competing interests

There are no conflicts of interest to declare.

3. Availability of data and material:

The datasets used or analysed during the current study are available from the corresponding author on reasonable request.

4. Code availability:

$\mathrm{N} / \mathrm{A}$

5. Authors' contributions

Zhi-Xin Bai: conceptualization, data curation, formal analysis, investigation, methodology, writing-original draft

Cheng-Lu Jiang: formal analysis, investigation, methodology, writing-review and editing

Sheng-Hai Zhu: investigation, methodology, writing-review and editing

Mi Zhong: investigation, methodology, writing-review and editing

Ming-Jian Zhang: conceptualization, funding acquisition, methodology, writing-

review and editing

Fu-Sheng Liu: conceptualization, methodology, writing-review and editing

Bin Tang: methodology, software, writing-review and editing

Qi-Jun Liu: conceptualization, resources, writing-review and editing

Xiang-Hui Chang: conceptualization, investigation, methodology, project administration, supervision, writing-review and editing

\section{References}

[1] A.F. Schuch, R.L. Mills, Crystal Structures of the Three Modifications of Nitrogen 14 and Nitrogen 15 at High Pressure, J. Chem. Phys. 52 (1970) 6000. 
[2] M. Hanfland, M. Lorenzen, C. Wassilew-Reul, F. Zontone, Structures of Molecular Nitrogen at High Pressures, Rev. High Press. Sci. Technol. 7 (1998) 787.

[3] R.L. Mills, Bart Olinger, D.T. Cromer, Structures and phase diagrams of $\mathrm{N}_{2}$ and

CO to 13 GPa by x-ray diffraction, J. Chem. Phys. 84 (1986) 2837.

[4] W.E. Streib, T.H. Jordan, W.H. Lipscomb, Single-Crystal X-Ray Diffraction Study of $\beta$ Nitrogen, J. Chem. Phys. 37 (1962) 2962.

[5] D. Schiferl, D.T. Cromer, R.R. Ryan, A.C. Larson, R. Lesar, R.L. Mills, Structure of $\mathrm{N}_{2}$ at $2.94 \mathrm{GPa}$ and 300 K, Acta Cryst. C39 (1983) 1151.

[6] H. Olijnyk, High pressure x-ray diffraction studies on solid $\mathrm{N}_{2}$ up to $43.9 \mathrm{GPa}$, J. Chem. Phys. 93 (1990) 8968.

[7] D.T. Cromer, R.L. Mills, D. Schiferl, L.A. Schwalbe, The Structure of $\mathrm{N}_{2}$ at 49 kbar and $299 \mathrm{~K}$, Acta Cryst. B37 (1981) 8.

[8] G.W. Stinton, I. Loa, L.F. Lundegaard, M.I. McMahon, The crystal structures of $\delta a n d ~ \delta^{\star}$ nitrogen, J. Chem. Phys. 131 (2009) 104511.

[9] M.I. Eremets, A.G. Gavriliuk, I.A. Trojan, D.A. Dzivenko, R. Boehler, Single-bonded cubic form of nitrogen, Nat. Mater. 3 (2004) 558.

[10] M. Pu, S. Liu, L. Lei, F. Zhang, L.H. Feng, L. Qi, L.L. Zhang, Raman study of pressure-induced dissociative transitions in nitrogen, Solid State Commun. 298 (2019) 113645.

[11] M.J. Lipp, J. Park Klepeis, B.J. Baer, H. Cynn, W.J. Evans, V. lota, C.S. Yoo, Transformation of molecular nitrogen to nonmolecular phases at megabar pressures by direct laser heating, Phys. Rev. B 76 (2007) 014113.

[12] M.I. Eremets, A.G. Gavriliuk, I.A. Trojan, Single-crystalline polymeric nitrogen, Appl. Phys. Lett. 90 (2007) 171904.

[13] D. Tomasino, M. Kim, J. Smith, C.S. Yoo, Pressure-Induced Symmetry-Lowering Transition in Dense Nitrogen to Layered Polymeric Nitrogen (LP-N) with Colossal Raman Intensity, Phys. Rev. Lett. 113 (2014) 205502.

[14] D. Laniel, G. Geneste, G. Weck, M. Mezouar, P. Loubeyre, Hexagonal Layered Polymeric Nitrogen Phase Synthesized near 250 GPa, Phys. Rev. Lett. 122 (2019) 066001.

[15] L. Lei, Q.Q. Tang, F. Zhang, S. Liu, B.B. Wu, C.Y. Zhou, Evidence for a New Extended Solid of Nitrogen, Chin. Phys. Lett. 37 (2020) 068101. 
[16] M.J. Greschner, M. Zhang, A. Majumdar, H.Y. Liu, F. Peng, J.S. Tse, Y.S. Yao, A New Allotrope of Nitrogen as High-Energy Density Material, J. Phys. Chem. 120 (2016) 2920.

[17] R. Turnbull, M. Hanfland, J. Binns, M. M. Canales, M. Frost, M. Marqués, R.T. Howie, E. Gregoryanz, Unusually complex phase of dense nitrogen at extreme conditions, Nat. Commun. 9 (2018) 4717.

[18] J.F. Li, Q.W. Jiang, Z.Q. Zhu, H.Y. Zhu, X.L. Wang, Cage-like salt with N-N single bonds, Europhys. Lett. 124 (2018) 67004.

[19] J. Zhang, Z. Zeng, H.Q. Lin, Y. L. Li, Pressure-induced planar $\mathrm{N}_{6}$ rings in potassium azide, Sci. Rep. 4 (2014) 4358.

[20] A.A. Adeleke, M.J. Greschner, A. Majumdar, B. Wan, H.Y. Liu, Single-bonded allotrope of nitrogen predicted at high pressure, Phys. Rev. B 96 (2017) 224104.

[21] S.V. Bondarchuk, B.F. Minaevab, Super high-energy density single-bonded trigonal nitrogen allotropea chemical twin of the cubic gauche form of nitrogen, Phys. Chem. Chem. Phys. 19 (2017) 6698.

[22] Y.W. Li, X.L. Feng, H.Y. Liu, J. Hao, S.A.T. Redfern, W.W. Lei, D. Liu, Y.M. Ma, Route to high-energy density polymeric nitrogen t-N via He-N compounds, Nat. Commun. 9 (2018)722.

[23] X.L. Wang, Y.C. Wang, M.S. Miao, X. Zhong, J. Lv, T. Cui, J.F Li, L. Chen, C.J. Pickard, YM. Ma, Cagelike Diamondoid Nitrogen at High Pressures, Phys. Rev. Lett. 109 (2012)175502.

[24] J. C. Raich, R. L. Mills, a-y Transition in Solid Nitrogen and Carbon Monoxide at High Pressnre, J. Chem. Phys. 55 (1971) 1811.

[25] C. Mailhiot, L.H. Yang, A.K. McMahan, Polymeric nitrogen, Phys. Rev. B 46 (1992) 14419.

[26] D. Plašienka, R. Martoňák, Transformation pathways in high-pressure solid nitrogen: From molecular $\mathrm{N}_{2}$ to polymeric cg-N, J. Chem. Phys. 142 (2015) 094505.

[27] Y. Akahama, D. Ishihara, H. Yamashita, H. Fujihisa, N. Hirao, Y. Ohishi, Phase stability and magnetic behavior of hexagonal phase of $\mathrm{N}_{2}-\mathrm{O}_{2}$ system with kagome lattice under high pressure and low temperature, Phys. Rev. B 94 (2016) 064104.

[28] M.F. Pu, L. Li, Polarization Symmetry Breaking in Nitrogen under High Pressure, (2018).

[29] C.L. Jiang, W. Zeng, Y.D. Gan, F.S. Liu, B. Tang, Q.J. Liu, Structural softening of solid nitrogen under pressure by first-principles calculations, J. Phys. Chem. Solids 146 (2020) 109616.

[30] J.P. Perdew, K. Burke, M. Ernzerhof, Generalized Gradient Approximation Made Simple, Phys. Rev. Lett. 77 (1996) 3865-3868. 
[31] P. Hohenberg, W. Kohn, Generalized Gradient Approximation Made Simple, Phys. Rev. 136 (1964) B864.

[32] W. Kohn, L.J. Sham, Self-Consistent Equations Including Exchange and Correlation Effects, Phys. Rev. 140 (1965) A1133.

[33] S.J. Clark, M.D. Segall, C.J. Pickard, P.J. Hasnip, M.I.J. Probert, K. Refson, M.C. Payne, First principles methods using CASTEP, Z. Kristallogr. 220 (2005) 567-570.

[34] J.P. Perdew, A. Ruzsinszky, G.I. Csonka, O.A. Vydrov, G.E. Scuseria, L.A. Constantin, X.L Zhou, K. Burke, Restoring the Density-Gradient Expansion for Exchange in Solids and Surfaces, Phys. Rev. Lett. 100 (2008) 136406.

[35] D. Antonangeli, G. Morard, L. Paolasini, G. Garbarino, C.A. Murphy, E. Edmund, F. Decremps, G. Fiquet, A. Bosak, M. Mezouar, Y.W. Fei, Sound velocities and density measurements of solid hcp-Fe and hcp-FeSi (9wt.\%) alloy at high pressure: Constraints on the Si abundance in the Earth's inner core, Earth Planet. Sc. Lett. 482 (2018) 446.

[36] T. Sakamaki, E. Ohtani, H. Fukui, S. Kamada, S. Takahashi, T. Sakairi, A. Takahata, T. Sakai, S. Tsutsui, D. Ishikawa, R. Shiraishi, Y. Seto, T. Tsuchiya, A.Q.R. Baron, Constraints on Earth's inner core composition inferred from measurements of the sound velocity of hcp-iron in extreme conditions, Sci. Adv. 2 (2016) 1500802.

[37] G. VSin'ko, N.A. Smirnov, On elasticity under pressure, J. Phys. Condens. Matter 16 (2004) 5101.

[38] B. Martorell, L. Vocadlo, J. Brodholt, I.G. Wood, Strong Premelting Effect in the

Elastic Properties of hcp-Fe Under Inner-Core Conditions, Science 342 (2013) 466.

[39] J. Belak, R. LeSar, R.D. Etters, Calculated thermodynamic properties and phase transitions of solid N2 at temperatures $0 \leq T \leq 300 \mathrm{~K}$ and pressures $0 \leq \mathrm{P} \leq 100 \mathrm{GPa}$, J. Chem. Phys. 92 (1990) 5430.

\section{Figures}



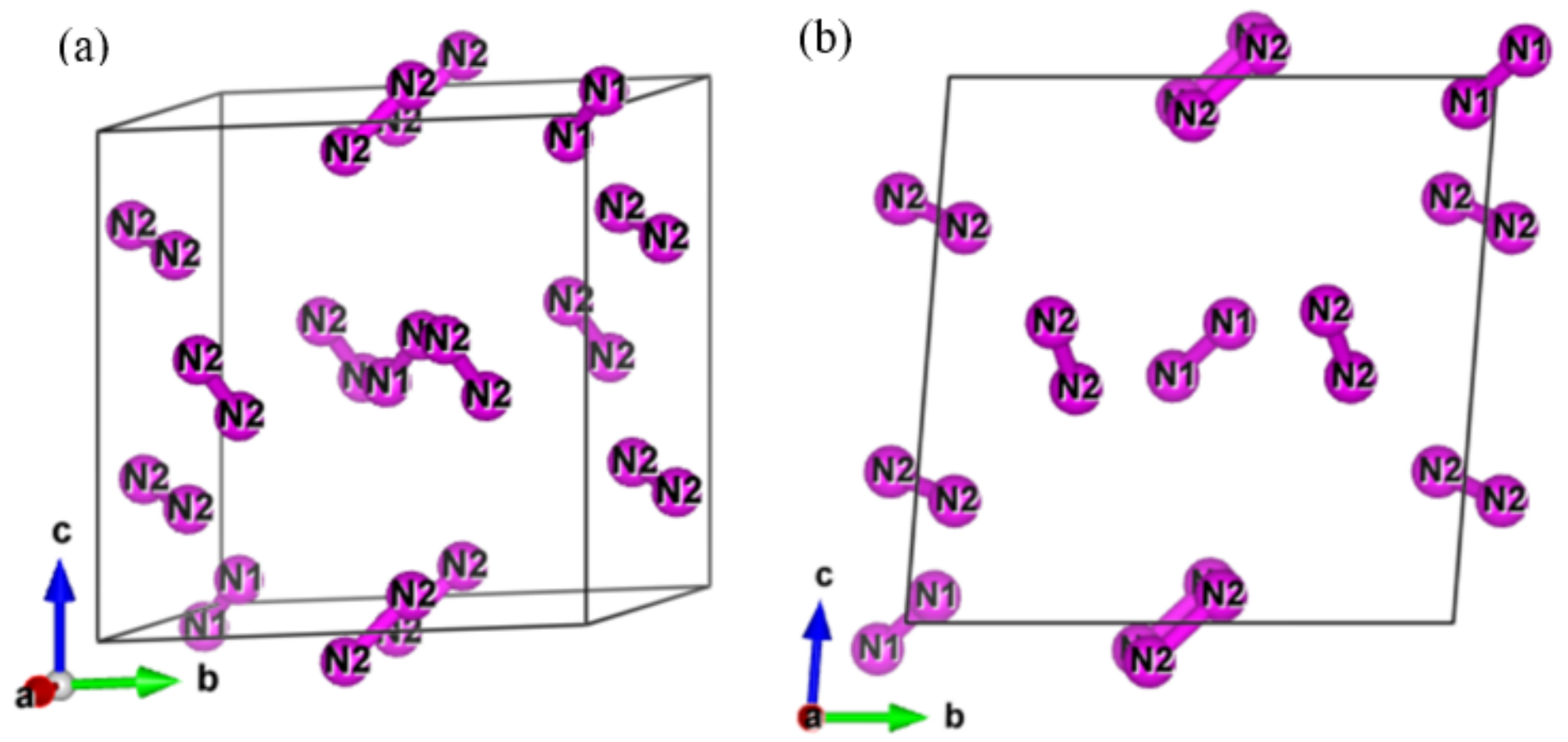

Figure 1

Computational model of solid nitrogen, N1 (0, 0, 0.0495), N2 $(0.2731,0.2127,0.2828)$.

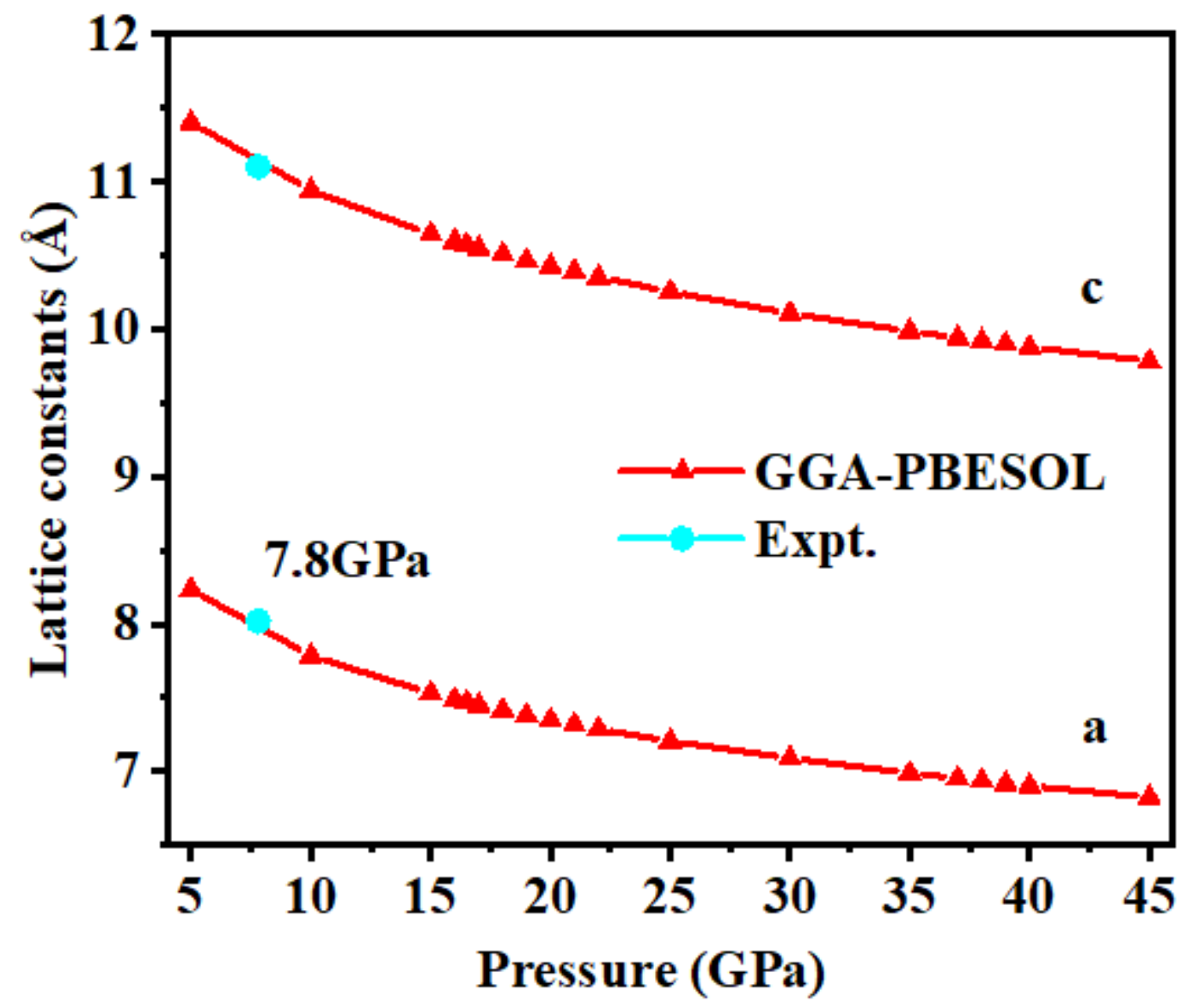


Figure 2

The calculated lattice constants of solid nitrogen under pressure.

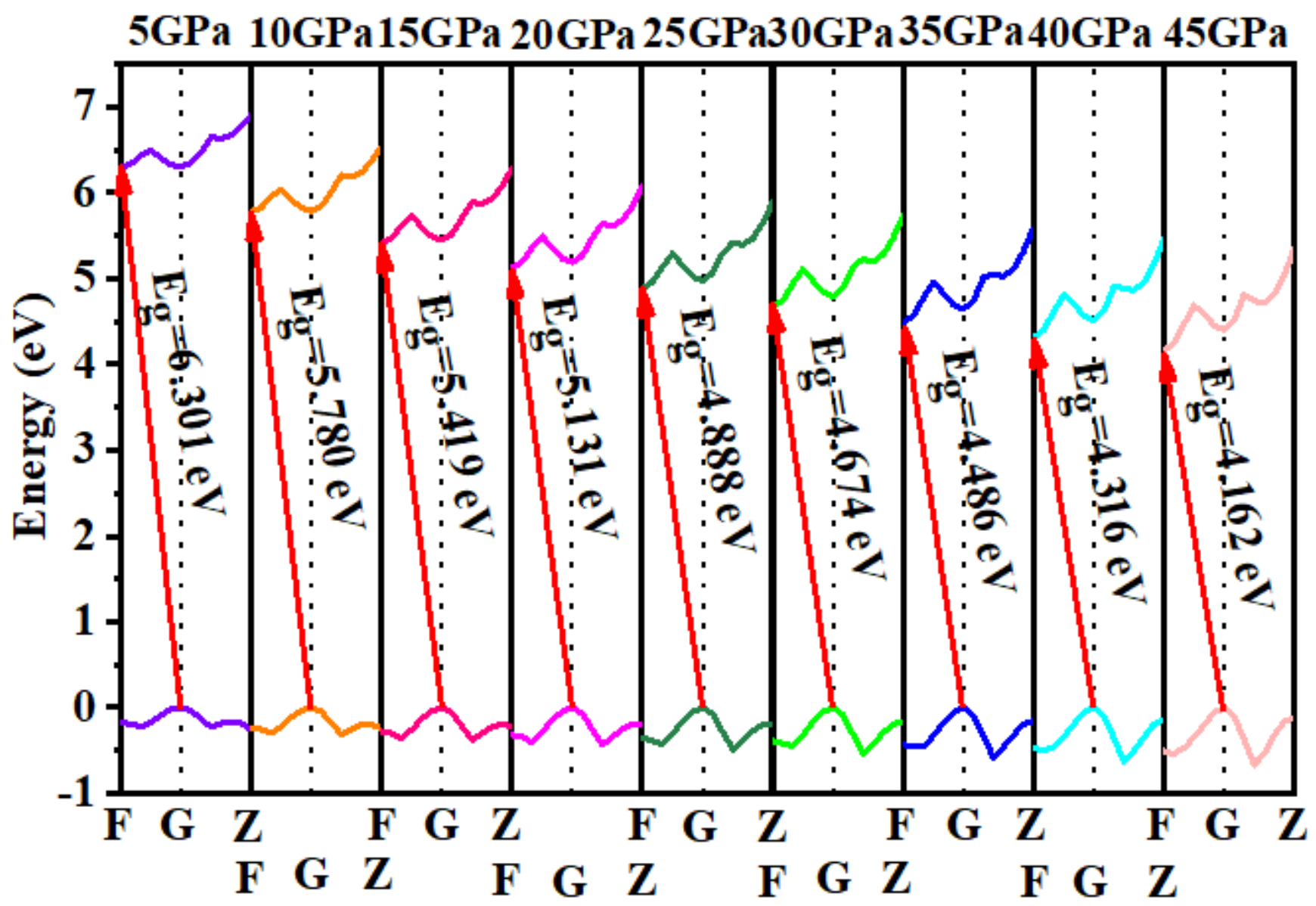

Figure 3

The band structures of solid nitrogen under pressure. 


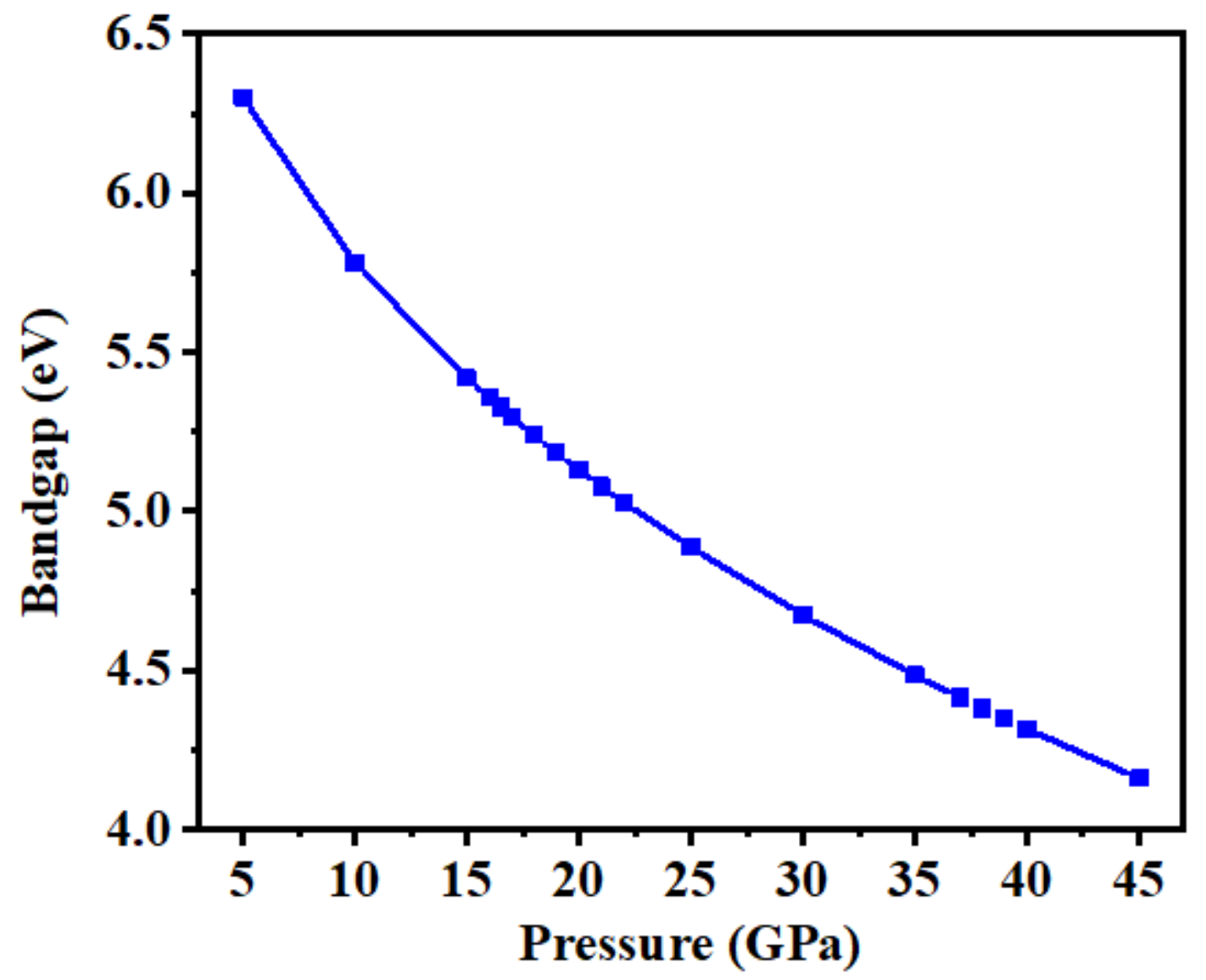

Figure 4

The calculated bandgaps of solid nitrogen under pressure. 

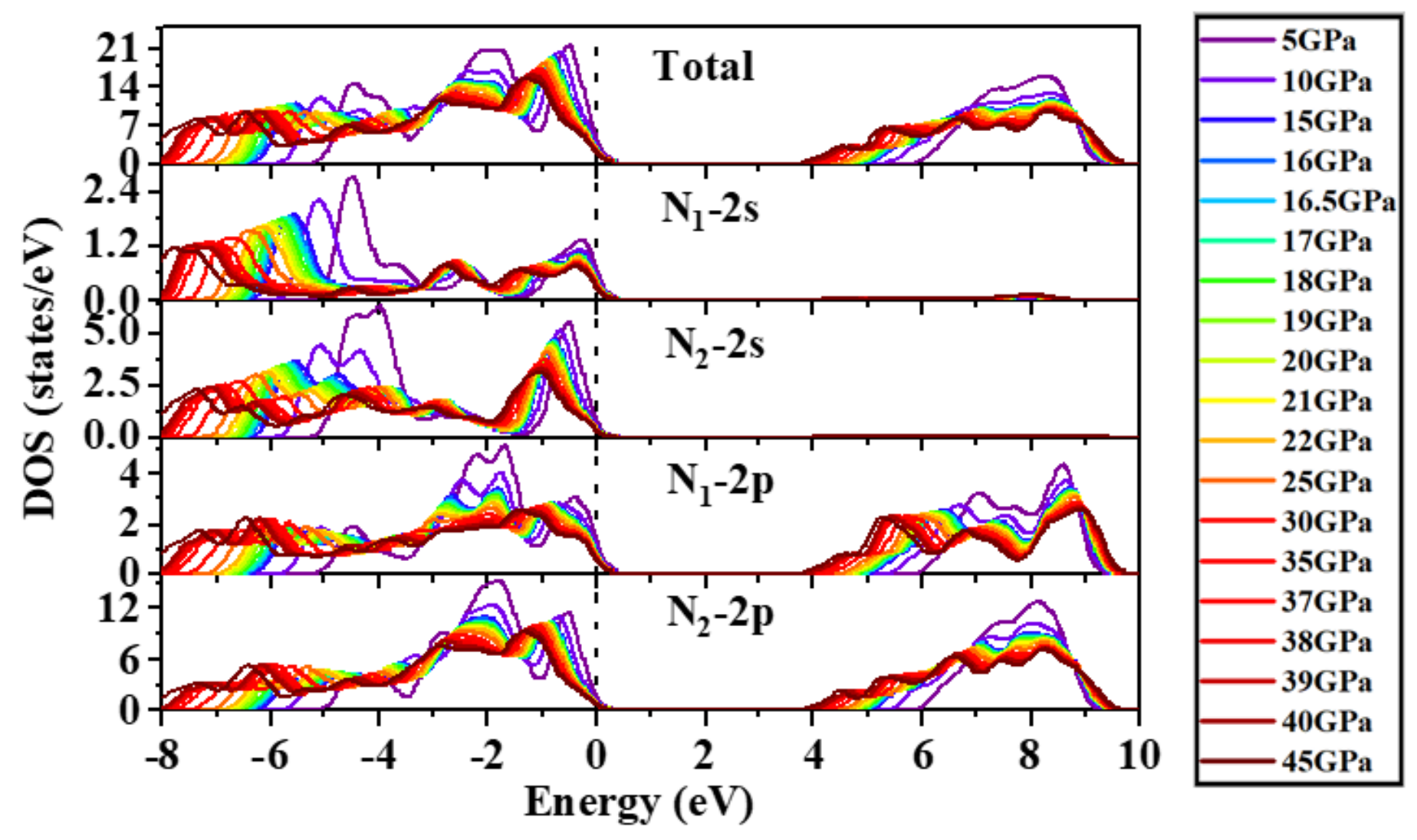

Figure 5

The total and partial densities of states of solid nitrogen under pressure.
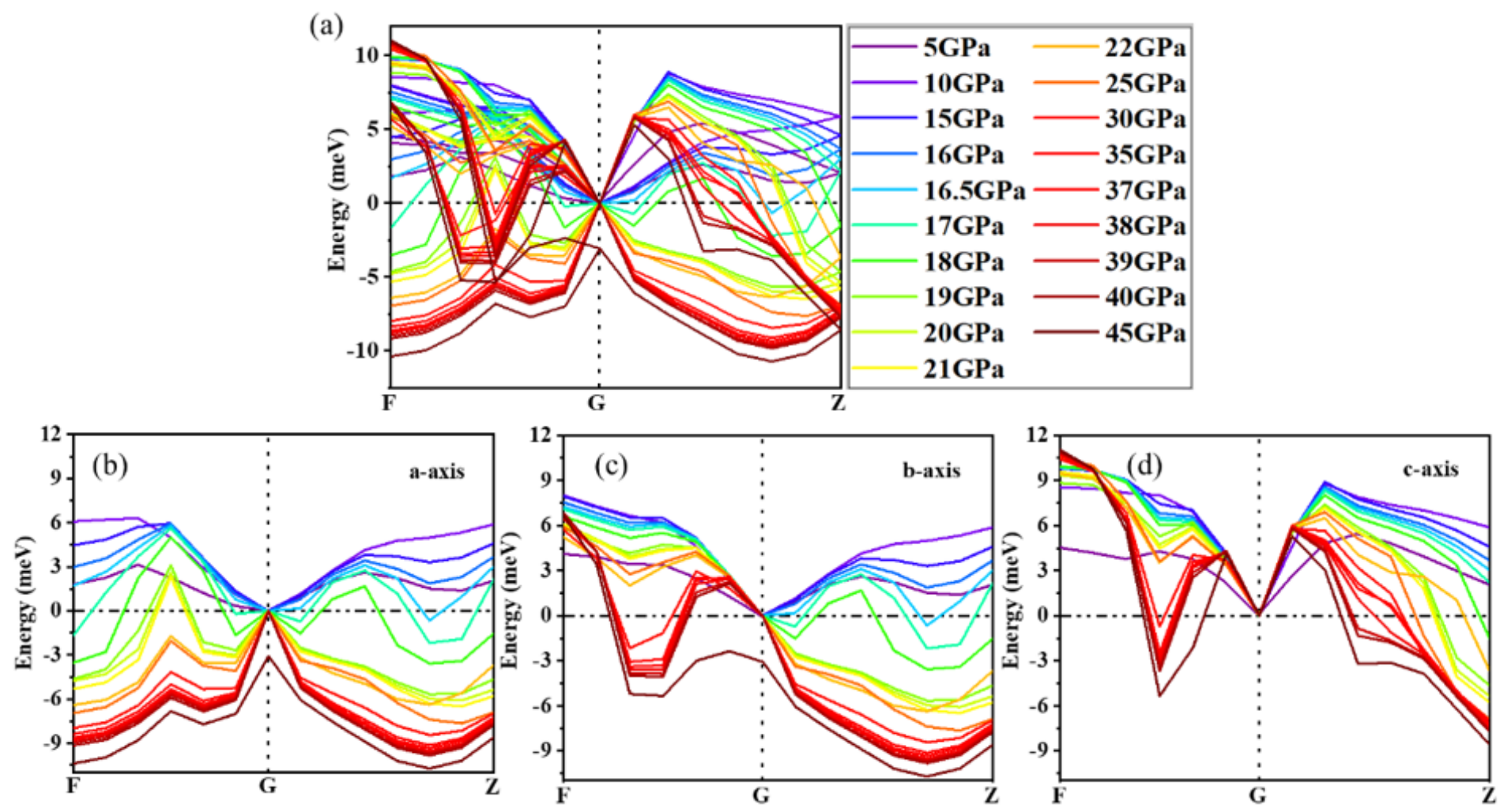

Figure 6 
The acoustic phonons of solid nitrogen under pressure in (a) a,b,c-axis, (b) a-axis, (c) b-axis and (d) c-axis, $F(0.5,0.5,0), G(0,0,0), Z(0.5,0.5,0.5)$

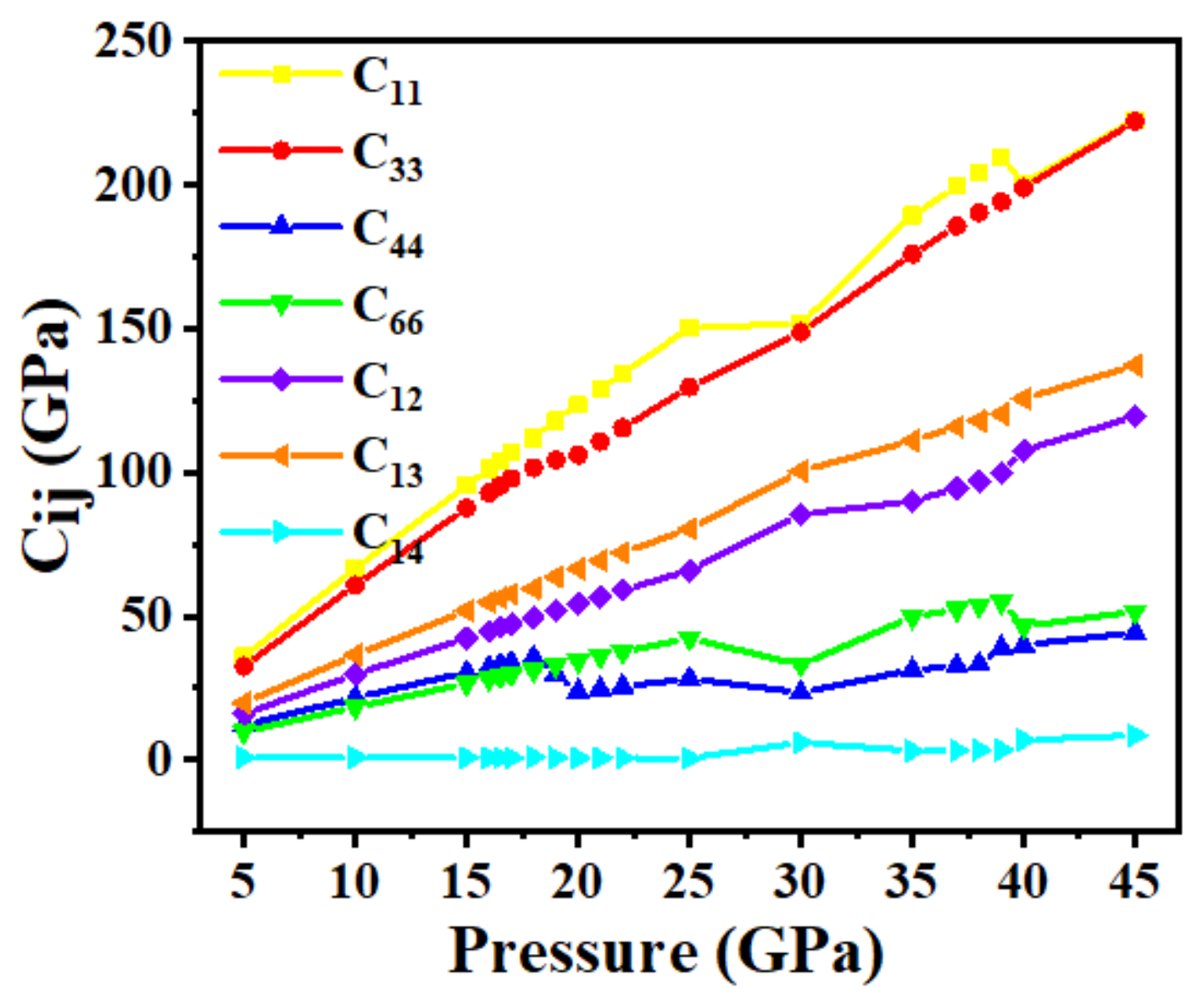

Figure 7

Calculated elastic constants $\mathrm{Cij}$ of solid nitrogen under pressure 


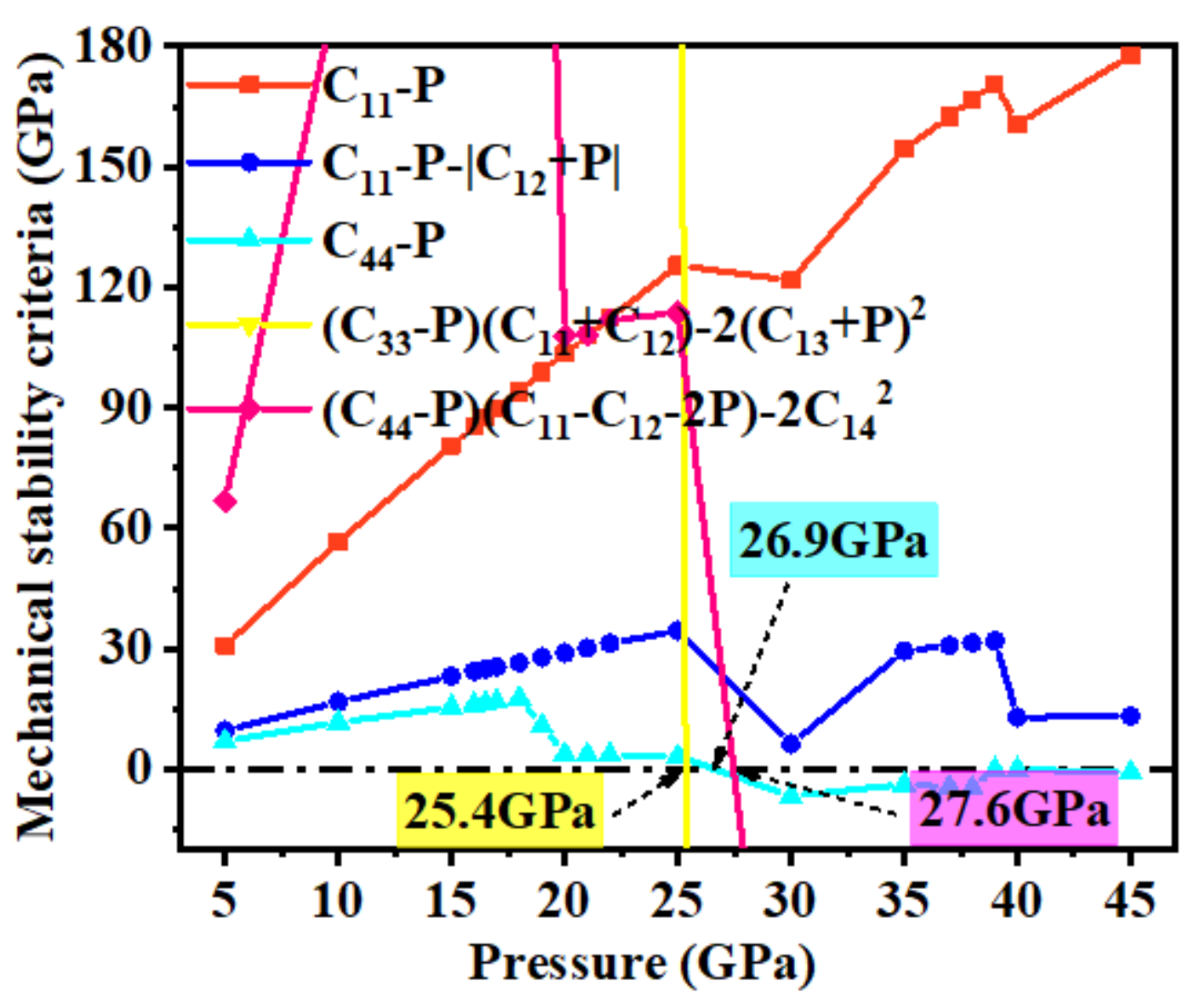

Figure 8

The mechanical stability criteria of solid nitrogen under pressure. 


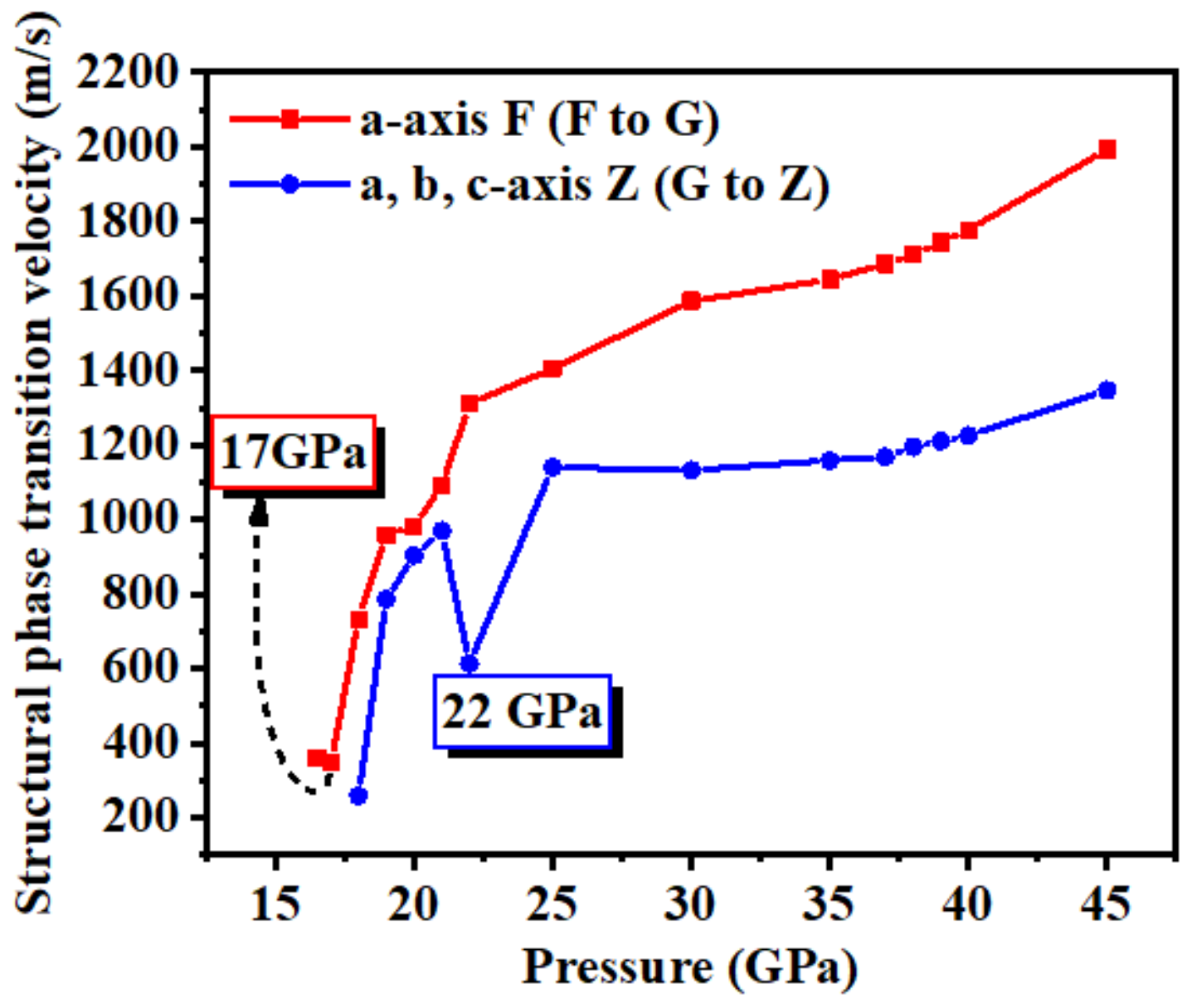

Figure 9

The structural phase transition velocities of solid nitrogen under pressure. 


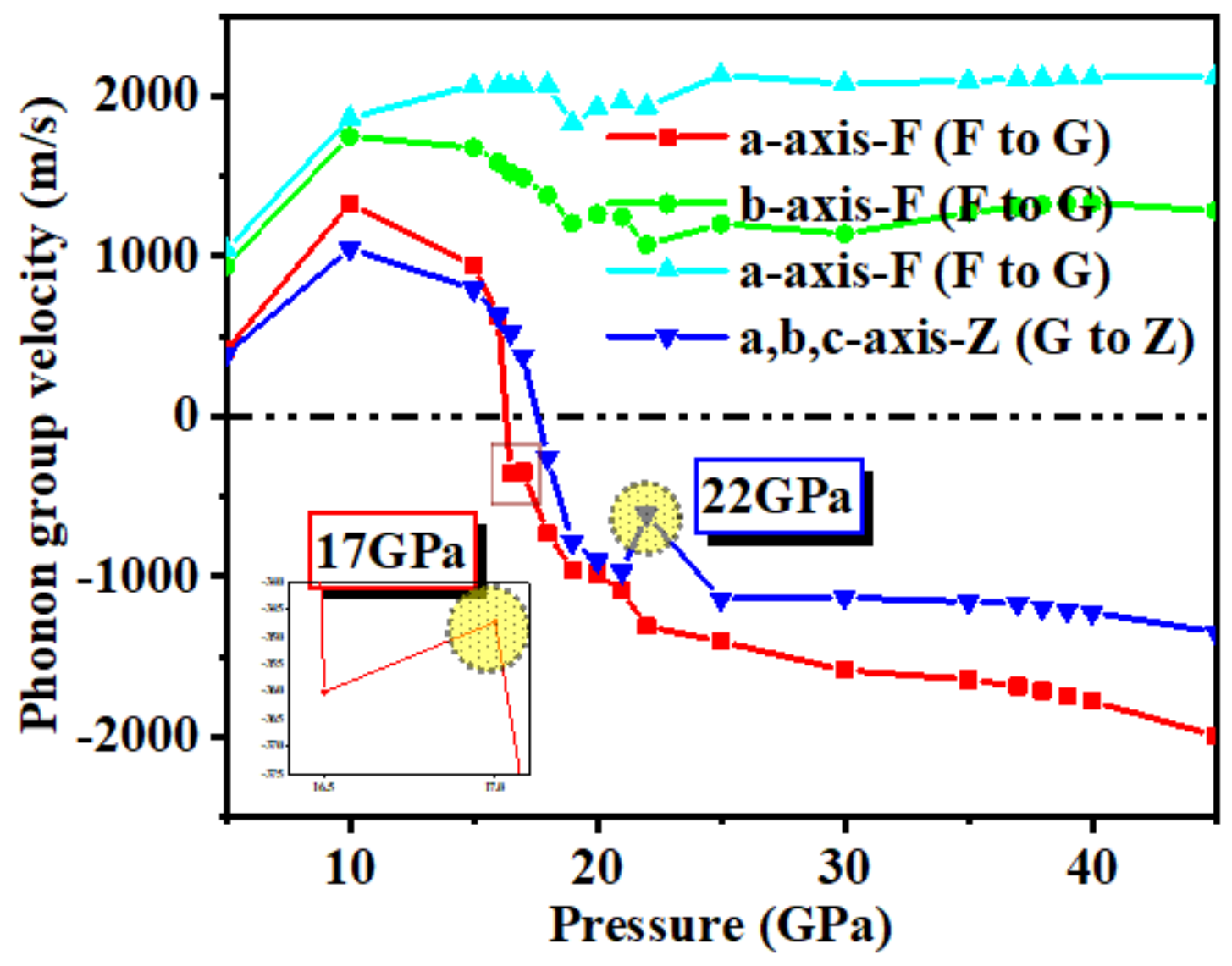

Figure 10

The phonon group velocities of rhombohedral solid nitrogen under pressure. 